\title{
Navelbine and Capecitabine (NavCap) versus NavCap Followed by Weekly Docetaxel as First-Line Treatment for Patients with HER-2 Negative Metastatic Breast Cancer
}

\author{
Samia A. Ali, Hanan G. Mostafa, Mohamed-AlaaEldeen H. Mohamed, Noha A. Mohamed \\ Department of Clinical Oncology and Nuclear Medicine, Faculty of Medicine, Assuit University, Egypt
}

\begin{abstract}
Background and Aim: There is an ongoing effort to optimize the management of HER-2 negative metastatic breast cancer (MBC). The aim of this study was to assess the treatment outcome of vinorelbine combines with capecitabine (NavCap) compared to the same combination followed by sequential single agent docetaxel in patients with HER-2 negative MBC.

Patients and Methods: Patients with HER-2 negative MBC previously treated with anthracycline in the adjuvant setting were enrolled in this prospective phase II study. Patients received vinorelbine $25 \mathrm{mg} / \mathrm{m}^{2}$ on day 1 and 8 combined with capecitabine $825 \mathrm{mg} / \mathrm{m}^{2}$ twice daily on days 1 to 14 (NavCap) every 3 weeks for 4 cycles. Patients with complete response (CR), partial response (PR) and stable disease (SD) were randomized to two arms. Arm 1 received another 4 cycles of NavCap and arm 2 received docetaxel 25mg/ $\mathrm{m}^{2}$ weekly for 12 weeks.

Results: From March 2012 to March 2014, 35 patients were enrolled in the study. Thirty-one patients were randomized to arm 1 (16 patients) and arm 2 (15 patients). The overall response rate was 50\% and 60\% in arms 1 and 2, respectively. With a median follow-up of 15 month, the median time to tumor progression was 13 and 12 months and the median survival were 17 and 16 months for arms 1 and 2, respectively. The most frequent treatment related toxicities in arm 1 were: grade 3- 4 neutropenia $(12.5 \%)$, anemia $(6.25 \%)$ and grade 2 nausea and vomiting (12.5\%). In arm 2, grade $3-4$ neutropenia (6.7\%), anemia (6.7\%) and grade 2 alopecia $(13.3 \%)$ were reported.

Conclusion: Both NavCap and NavCap followed by docetaxel schedules appear to be effective and welltolerated regimens as first line treatment for Egyptian HER-2 negative MBC.
\end{abstract}

Key words: Her-2 Negative, Metastatic Breast Cancer, First-line, Vinorelbine-capecitabine, Sequential docetaxel Corresponding Author: Hanan G. Mostafa

E-mail: mostafahanan36@yahoo.com

\section{INTRODUCTION}

Excluding cutaneous malignancies, breast cancer is the most common malignancy. It accounts for nearly one in three cancers diagnosed among American females and the second leading cause of cancer deaths worldwide ${ }^{1,2}$.

The prevalence of metastases within the 5-year postsurgery period is $20 \%$ among patients with lymph nodenegative breast cancer and 50- 60\% among those with lymph node-positive ${ }^{3}$. Since metastatic breast cancer (MBC) is still incurable, the objectives are overall survival (OS) extension, tumor progression delay and quality of life improvement ${ }^{4}$.

Nowadays anthracycline are frequently included in the adjuvant management of breast cancer. As a result, many of the patients who present with recurrent breast cancer have already received anthracyclines ${ }^{5}$. The palliative intent of systemic chemotherapy in $\mathrm{MBC}$ patients should be respected when choosing a strategy for its administration. In addition the decision to administer combination chemotherapy should be guided by the performance status of the patient as well as the need to control visceral metastases ${ }^{6}$.

Vinorelbine is a microtubule inhibitor which is effective in the treatment of MBC patients with exposure or resistance to anthracyclines and taxanes ${ }^{7}$. Capecitabine is a precursor of 5-deoxy-fluorouridine that is converted in tumor tissue to 5-fluorouracil ${ }^{8}$. It is administered orally and can be rapidly absorbed, with low toxicity9.

Intravenous vinorelbine plus capecitabine to treat MBC patients resulted in a response rate ranging from 49 to $70 \%$ in phase II studies with acceptable toxicities ${ }^{10,11}$.

Among the effective treatment options in $\mathrm{MBC}$ is single agent taxanes, especially in patients who received anthracycline-based regimen only as adjuvant ${ }^{6}$. 
Kasr-El-Aini Journal Of Clinical Oncology And Nuclear Medicine

\begin{tabular}{ll|ll} 
Vol. 11 No. $1-2$ & 2015 & Ali S.A. et al
\end{tabular}

There is good evidence that taxane-based regimens improve OS, time to progression as well as response rate when used either as first line or as further lines in MBC patients ${ }^{12}$. In a phase II randomized study that compared weekly docetaxel to 3-weekly administration in $\mathrm{MBC}$ found a more or less similar efficacy in terms of response rate and time to progression. However, the toxicity profile was in favor of weekly docetaxe ${ }^{13}$.

In a phase II pilot study, Ghosn and his colleagues evaluated the response to the sequential use of vinorelbine $\left(25 \mathrm{mg} / \mathrm{m}^{2}\right.$, days $\left.1 \& 8\right)$ and capecitabine $\left(825 \mathrm{mg} / \mathrm{m}^{2}\right.$ twice daily, days 1 -14) every 3 weeks (NavCap) for 4 cycles followed by 12 consecutive weeks of docetaxel (25mg/ $\mathrm{m}^{2}$ ) as first-line treatment for MBC. The results were promising with prolonged time to progression and OS and acceptable toxicity ${ }^{14}$. The encouraging results of that study had led Ghosn and his colleagues to conduct a further phase II randomized trial to compare 8 cycles of NavCap to 4 cycles of NavCap followed by weekly docetaxel ${ }^{15}$. Both regimens resulted in more or less similar efficacy and manageable toxicity.

The present study was undertaken to further compare NavCap to Nav-Cap followed by weekly docetaxel as first line therapy in Egyptian patients with HER-2 negative $\mathrm{MBC}$.

\section{PATIENTS AND METHODS}

This is a phase II prospective study for patients with MBC who presented to the Clinical Oncology Department, Assiut University Hospital. The protocol of the study was approved by the ethics committee of the Faculty of Medicine, Assiut University, Egypt.

\section{Selection of patients}

Inclusion criteria included histologically confirmed breast adenocarcinoma, documented metastatic disease, estimated life expectancy of $>12$ weeks, adequate bone marrow reserve, normal liver and renal functions, Eastern Cooperative Oncology Group (ECOG) performance status $<3$ and at least one measurable lesion by imaging. HER-2 status was assessed by immunohistochemistry (IHC). The disease free interval should be at least 12 months after adjuvant anthracycline and/or taxanes. Prior 5-fluororacil was allowed in the adjuvant setting and hormonal therapy for metastatic disease should have been stopped at patient inclusion. Written informed consent was obtained from all patients.

Exclusion criteria included previous chemotherapy for metastatic disease, previous treatment with a vinca alkaloid or capecitabine, peripheral neuropathy $\geq 2$ according to version 3 of the National Cancer Institute -Common Terminology criteria adverse events (NCI CTCAE v3.0), dysphagia or inability to swallow tablets, malabsorption syndrome, unstable diabetes, uncontrolled hypercalcemia, serious illness (e.g. cardiac disease or liver dysfunction), brain metastases, pregnancy or lactation and radiotherapy to measurable lesions. Previous radiotherapy to bone metastases was allowed but should have been completed for more than 4 weeks.

\section{Study evaluation}

Complete response (CR), partial response (PR), stable disease (SD) and progressive disease (PD) were defined according to Response Evaluation Criteria in Solid Tumors (RECIST). Overall response rate (ORR) is defined as the sum of $\mathrm{CR}$ and PR, while clinical benefit rate $(\mathrm{CBR})$ is the sum of ORR and SD.

The primary endpoint was the overall response rate (CR plus PR) for $>3$ months. Secondary endpoints included time to progression (TTP), OS and the toxicity profile.

All patients were subjected to full medical history and physical examination including ECOG performance status grading and measurements of palpable or visual tumor lesions. Laboratory investigation included complete blood picture, liver and kidney function tests at day 1 of each cycle of NavCap and every 3 doses of weekly docetaxel. Radiological studies included chest $\mathrm{x}$-ray, computed tomography (CT) or magnetic resonant imaging (MRI) of chest and abdomen and bone scan. This was done before the study entry, after two cycles of the NavCap protocol, 6 weeks of weekly docetaxel and at the end of therapy in each arm. It may also be done at any time to document progression by physical examination and disease evaluation.

Response evaluation using the RECIST was done after 4 NavCap cycles and at the end of therapy and every 3 months after. Adverse events were recorded every cycle and graded according to the NCI-CTCAE v3.0.

\section{Study design and treatment}

All patients received 4 cycles of the NavCap regimen which consisted of vinorelbine $25 \mathrm{mg} / \mathrm{m}^{2}$ administered by rapid intravenous infusion on day 1 and 8 of a 21-day cycle, plus capecitabine $825 \mathrm{mg} / \mathrm{m}^{2}$ PO every 12 hours with a glass of water within 30 minutes of a meal for 14 days every 3 weeks.

Patients with CR, PR and SD were randomized by simple randomization into 2 arms, arm 1 and arm 2. Arm 1 included NavCap with the same schedule for further 
4 cycles. Arm 2 patients received docetaxel $25 \mathrm{mg} / \mathrm{m}^{2}$ weekly for 12 weeks, over one hour intravenous infusion with $4 \mathrm{mg}$ of dexamethasone on the morning and evening of the date of administration.

If grade 3- 4 hematological or non-hematological toxicities occurred, a $25 \%$ dose reduction in the next cycle was done and maintained during all following cycles. If grade 3 hand-foot syndromes occurred, treatment was delayed for one week. If toxicity was not resolved, capecitabine was resumed with $25 \%$ dose reduction.

Patients who progressed before randomization received $2^{\text {nd }}$ line chemotherapy.

\section{Statistical analysis}

Data is expressed by mean, standard deviation, numbers and percentage. Means were compared using the student's T-test. The Chi square test was used to evaluate percentage differences between both arms.

TTP was calculated from the date of the start of treatment to the date of the first documentation of disease progression or death. OS was calculated from the time starting treatment to death due to any cause with censoring at the last date known alive. The median TTP and OS were estimated with the Kaplan-Meier method and compared by log-rank test.

A $p$-value $<0.05$ was considered statistically significant. All statistical analyses were analyzed by computer program using SPSS 21 “SPSS, Inc., Chicago, IL”.

\section{RESULTS}

Thirty-five female patients were enrolled in the study from March 2012 to March 2014. All eligible patients received 4 cycles of NavCap. Four patients progressed after 4 cycles of NavCap and they were excluded from randomization.

The characteristics of patients before randomization are detailed in Table 1.

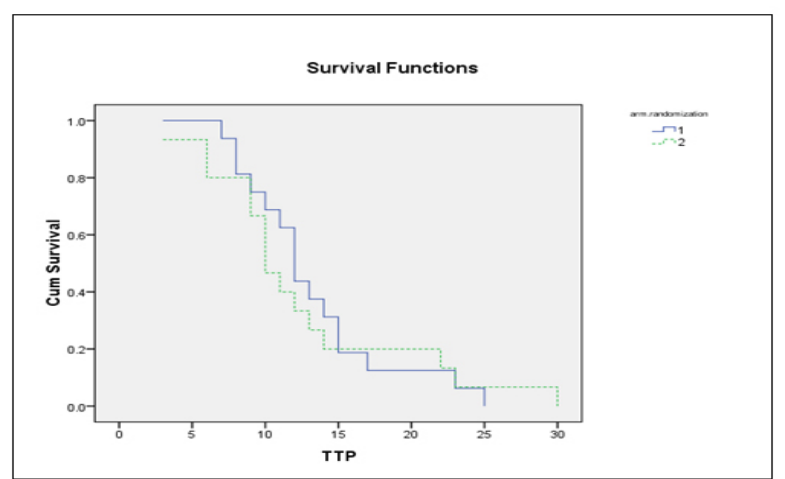

Figure 1: Kaplan-Meier curves of time to progression (TTP) according to the treatment arm.
The response to 4 cycles of NavCap and before randomization is illustrated in Table 2.

None of the patients achieved complete remission and the overall response rate (PR plus $\mathrm{SD}$ ) was $89 \%$. The 4 patients who had $\mathrm{PD}$ received $2^{\text {nd }}$ line chemotherapy.

Patients' characteristics after randomization were analyzed (Table 3 ).

Comparing arm 1 with arm 2, there was no significant difference in age, performance status, grade, hormonal receptors, adjuvant hormonal therapy or number and sites of metastases.

As regard treatment outcome after randomization, there was no significant difference between both arms regarding ORR $(\mathrm{p}=0.762)$. The $\mathrm{CBR}$ was almost similar in both arms, $75 \%(12 / 16)$ in arm 1 and $73 \%(11 / 15)$ in arm 2 (Table 4).

The TTP and OS curves are illustrated in Figures 1 and 2 .

The TTP did not differ significantly between the 2 arms $(\mathrm{p}=0.72)$. The mean TTP was 13.19 and 12.53 months in arms 1 and 2, respectively. Similarly, the OS did not differ significantly $(\mathrm{p}=0.77)$ and the mean was 18.75 months in arm 1 and 18.13 months in arm 2 .

The treatment-related toxicities are shown in Table 5.

In both arms the most frequent treatment-related adverse events were hematological toxicities. None of the non-hematological toxicities was of grade 34-. The most common non-hematological toxicities in arm 1 were nausea and vomiting, hand-foot syndrome and diarrhea, while in arm 2 were mucositis, hand-foot syndrome and peripheral neuropathy.

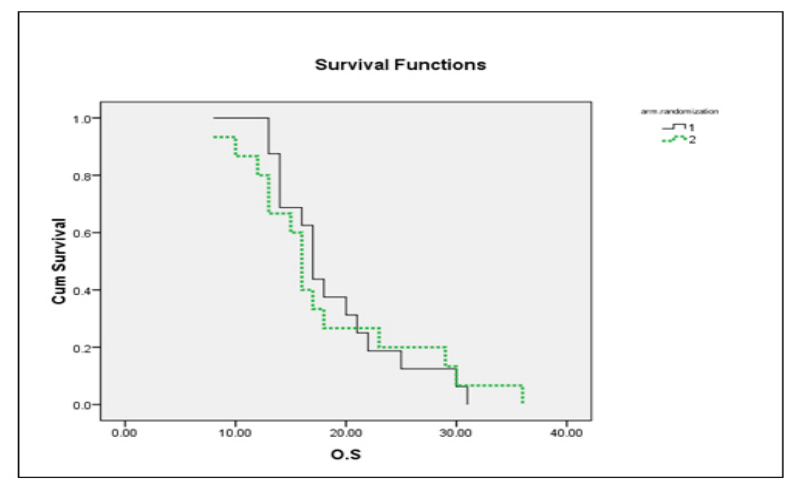

Figure 2: Kaplan-Meier curves of overall survival (OS) according to the treatment arm 
Ali S.A. et al

Table 1: Patients characteristics before randomization

\begin{tabular}{|c|c|}
\hline Characteristic & No. $(\%)$ \\
\hline \multicolumn{2}{|l|}{ Age } \\
\hline Median (range) & $52(35-69)$ \\
\hline \multicolumn{2}{|c|}{$\begin{array}{l}\text { Eastern Cooperative Oncology Group (ECOG) } \\
\text { performance status }\end{array}$} \\
\hline 0 & $4(11.4)$ \\
\hline 1 & $24(68.6)$ \\
\hline 2 & $7(20)$ \\
\hline \multicolumn{2}{|l|}{ Grade } \\
\hline II & $27(77.1)$ \\
\hline III & $8(22.9)$ \\
\hline \multicolumn{2}{|l|}{ Hormone receptor status } \\
\hline Negative & $12(34.3)$ \\
\hline Positive & $23(65.7)$ \\
\hline \multicolumn{2}{|l|}{ Adjuvant hormonal therapy } \\
\hline No & $15(42.9)$ \\
\hline Yes & $20(57.1)$ \\
\hline \multicolumn{2}{|l|}{ No. of metastatic sites } \\
\hline 1 & $15(42.9)$ \\
\hline 2 & $16(45.7)$ \\
\hline$\geq 3$ & $4(11.4)$ \\
\hline \multicolumn{2}{|l|}{ Metastatic site } \\
\hline Bone and soft tissue & $21(60)$ \\
\hline Viscera (liver, lung) & $18(51.4)$ \\
\hline Other (distant lymph nodes) & $3(8.6)$ \\
\hline \multicolumn{2}{|l|}{ Disease-free interval (months) } \\
\hline Mean \pm standard deviation & $23.1 \pm 18.4$ \\
\hline Median & 18 \\
\hline
\end{tabular}

Table 2: The response of 35 patients with metastatic breast cancer to 4 cycles of vinorelbine-capecitabine (NavCap) combination before randomization

\begin{tabular}{ll}
\hline Response & No. (\%) \\
\hline Partial response & $15(42.9)$ \\
\hline Stable disease & $16(45.7)$ \\
\hline Progressive disease & $4(11.4)$ \\
\hline
\end{tabular}

Table 3: Characteristics of patients after allocation to treatment arm

\begin{tabular}{|c|c|c|}
\hline \multirow[t]{2}{*}{ Characteristic } & $\begin{array}{l}\text { Arm 1 } \\
(n=16)\end{array}$ & $\begin{array}{l}\text { Arm 2 } \\
(n=15)\end{array}$ \\
\hline & No. $(\%)$ & No. $(\%)$ \\
\hline \multicolumn{3}{|l|}{ Age } \\
\hline Median (range) & $52(47-69)$ & $45(35-69)$ \\
\hline \multicolumn{3}{|c|}{$\begin{array}{l}\text { Eastern Cooperative Oncology } \\
\text { Group (ECOG) performance status }\end{array}$} \\
\hline 0 & $3(18.8)$ & $1(6.7)$ \\
\hline 1 & $8(50)$ & $12(80)$ \\
\hline 2 & $5(31.2)$ & $2(13.3)$ \\
\hline \multicolumn{3}{|l|}{ Grade } \\
\hline II & $11(68.8)$ & $13(86.7)$ \\
\hline III & $5(31.2)$ & $2(13.3)$ \\
\hline \multicolumn{3}{|l|}{ Hormone receptor status } \\
\hline Negative & $6(37.5)$ & $5(33.3)$ \\
\hline Positive & $10(62.5)$ & $10(66.7)$ \\
\hline \multicolumn{3}{|c|}{ Adjuvant hormonal therapy } \\
\hline No & $8(50)$ & $5(33.3)$ \\
\hline Yes & $8(50)$ & $10(66.7)$ \\
\hline \multicolumn{3}{|l|}{ No. of metastatic sites } \\
\hline 1 & $7(43.8)$ & $8(53.4)$ \\
\hline 2 & $8(50)$ & $5(33.3)$ \\
\hline$\geq 3$ & $1(6.2)$ & $2(13.3)$ \\
\hline \multicolumn{3}{|l|}{ Metastatic site } \\
\hline Non-visceral & $13(81.3)$ & $11(73.3)$ \\
\hline Viscera (liver, lung) & $7(43.8)$ & $7(46.6)$ \\
\hline
\end{tabular}

Table 4: Treatment outcome of metastatic breast cancer patients after randomization to Navcap or docetaxel

\begin{tabular}{|c|c|c|}
\hline \multirow[t]{2}{*}{ Treatment outcome } & $\operatorname{Arm} 1(n=16)$ & $\operatorname{Arm} 2(n=15)$ \\
\hline & No. $(\%)$ & No. $(\%)$ \\
\hline Complete response (CR) & $2(12.5)$ & $2(13.3)$ \\
\hline Partial response (PR) & $7(43.5)$ & $7(46.7)$ \\
\hline Stable disease (SD) & $3(18.8)$ & $2(13.3)$ \\
\hline Progressive disease (PD) & $4(25)$ & $4(26.7)$ \\
\hline $\begin{array}{l}\text { Overall response rate (CR } \\
+\mathrm{PR})\end{array}$ & $9(56.3)$ & $9(60)$ \\
\hline $\begin{array}{l}\text { Clinical benefit rate }(\mathrm{CR}+ \\
\mathrm{PR}+\mathrm{SD})\end{array}$ & $12(75)$ & $11(73.3)$ \\
\hline
\end{tabular}

Table 5: Toxicities in both treatment arms.

\begin{tabular}{|c|c|c|c|c|}
\hline \multirow[t]{2}{*}{ Toxicity } & \multicolumn{2}{|l|}{ Grade 1 - 2} & \multicolumn{2}{|l|}{ Grade $3-4$} \\
\hline & $\operatorname{Arm~1}^{*}(n=16)$ & $\operatorname{Arm} 2^{*}(n=16)$ & $\operatorname{Arm~1*}^{*}(n=16)$ & $\operatorname{Arm} 2 *(n=16)$ \\
\hline \multicolumn{5}{|l|}{ Hematological } \\
\hline Anemia & $6(37.5)$ & $2(13.3)$ & $1(6.3)$ & $1(6.7)$ \\
\hline Neurtopenia & $2(12.5)$ & $1(6.7)$ & $2(12.5)$ & $1(6.7)$ \\
\hline
\end{tabular}

Non-Hematological

Nausea and vomiting

Diarrhea

Alopecia

Mucositis

Peripheral neuropathy

Hand-foot syndrome

\begin{tabular}{ccll}
$2(12.5)$ & 0 & 0 & 0 \\
\hline $1(6.3)$ & 0 & 0 & 0 \\
\hline 0 & $2(13.3)$ & 0 & 0 \\
\hline 0 & $1(6.7)$ & 0 & 0 \\
\hline 0 & $1(6.7)$ & 0 & 0 \\
\hline $2(12.5)$ & 0 & 0 & 0 \\
\hline
\end{tabular}

**Arm 1: 8 cycles NavCap; Arm 2: 4 cycles NavCap followed by weekly docetaxel 


\section{DISCUSSION}

With all efforts to optimize the management of patients with early breast cancer, almost one third of these patients will develop local or distant treatment failure. MBC continues to be an incurable disease and its prognosis remains dismal with a 5-year relative survival rate less than 30\% ${ }^{16-17}$.Consequently, the goal of MBC management is palliative with the aim of improving the quality of life and prolonging progression free survival and possibly OS. For MBC, the optimal management strategy should take into consideration multiple prognostic and predictive factors such as hormonal receptor status, HER-2 status, visceral metastases and response to previous therapy ${ }^{5}$.

Chemotherapy is still the mainstay of treatment of advanced breast cancer as it provides tumor shrinkage and noticeable clinical benefit ${ }^{18-19}$; hence it is accepted as a standard treatment for hormone-resistant rapidly progressive disease.

Anthracylines and taxanes are among the most effective agents as first line in MBC. However, they are used frequently in the adjuvant setting and many patients who develop MBC would have received these agents as adjuvants ${ }^{5}$.

The choice of the optimal treatment of MBC is further complicated by the unsolved debate about the superiority of combination chemotherapy versus sequential therapy ${ }^{20}$. In one review, poly-chemotherapy was superior regarding ORR, progression free survival and $\mathrm{OS}^{21}$

NavCap as first-line treatment for MBC patients was found to be an effective and tolerable regimen by Ghosn et al with an ORR of $70 \%{ }^{10}$. The outcome of MBC using NavCap followed by weekly docetaxel was encouraging with median TTP of 13 months and OS of 36 months $^{14}$. These results led Ghosn and his colleague to further evaluate NavCap versus NavCap followed by docetaxel. They found both regimens of similar efficacy with an ORR of $56 \%$ and $71 \%$, TTP of 10 and 12 months and OS of 35 and 37 months, respectively; with manageable toxicity for MBC patients ${ }^{15}$.

With these encouraging results, we investigated the efficacy and safety of NavCap in a population of Egyptian patients with MBC. The ORR was 56.3\% including two (12.5\%) CR and seven (43.5\%) PR after 8 cycles of NavCap (arm 1). The mean TTP was 13 months and the mean OS was 19 months.

The efficacy of NavCap as a first line for the treatment of MBC after failure of adjuvant anthracycline-based therapy was also evaluated by El-sadda et al, who reported an ORR of $60 \%$ with CR in $6(10 \%)$ patients after a median number of 7 cycles/patients (range $3-8)^{22}$. In that study, the median TTP and median OS were 14 and 23 months, respectively ${ }^{22}$. Another study by Tawfik et al was done to evaluate the efficacy and safety of all oral vinorelbine and capcitabine therapy in anthracycline \pm taxane pretreated HER-2 negative $\mathrm{MBC}^{23}$. They reported an ORR of $57 \%$, including CR in $11 \%$ and PR in $46 \%$, and the median TTP was 8.6 months and the median OS time was 27.2 months $^{23}$. The results of these two trials are comparable to ours except OS was higher due to longer follow-up.

In the current study, the toxicity profile of NavCap combination shows that the occurrence of grade 3 - 4 adverse events was limited to neutropenia in 2 $(12.5 \%)$ patients and anemia in one patient $(6.25 \%)$. Non-hematological grade $1-2$ toxicity as nausea and vomiting which occurred in two patients $(12.5 \%)$ and hand-foot syndrome grade $12-$ in $2(12.5 \%)$ patients. There was no grade 34/ non-hematological toxicity in the current study. These results are different from the results of the study done by Tawfik et $\mathrm{al}^{23}$, who reported a grade 4 neutropenia in $6(21.4 \%)$ patients, grade 3 nausea and vomiting in $2(7.1 \%)$ and $3(10.7 \%)$ patients respectively. Two (7.1\%) patients developed grade 3 hand and foot syndrome. This difference may be due to the higher dose of capcitabine $1000 \mathrm{mg} / \mathrm{m}^{2}$ in their study compared to $825 \mathrm{mg} / \mathrm{m}^{2}$ in the present study. On the other hand, the toxicity in our study was higher than that reported by El-sadda et $\mathrm{al}^{22}$, who reported grade 4 toxicities of neutropenia in 3 patients $(5 \%)$, and one patient $(1.7 \%)$ developed grade 3 hand and foot syndrome; while grade 2 anemia, neutropenia and diarrhea were reported in $2(3.3 \%), 3(5 \%)$ and 6 patients $(10.0 \%)$ respectively. The difference might be due to the lower number of cycles delivered in their study.

In the present study, $20(57 \%)$ patients had $\geq 2$ metastatic sites and $51.4 \%$ had visceral metastases. These characteristics justify the need for combination therapy and patients who showed a response with clinical benefit were allowed to continue either with the same combination or with weekly docetaxel. In the study done by Ghosn et a ${ }^{15}, 70 \%$ of patients had $>2$ involved organ sites, more than half of patients had visceral metastases and $19 \%$ of patients were stage IV at diagnosis.

Sequential single agent docetaxel was administered to one arm of patients after rapid controlling of symptoms and tumor burden. The choice of docetaxel 
Kasr-El-Aini Journal Of Clinical Oncology And Nuclear Medicine

\section{Vol. 11 |No. 1-2 2015}

rather than paclitaxel is based on the evidence that showed its superiority in the treatment of $\mathrm{MBC}^{24}$.

The recommendations by international guidelines advised the use of sequential mono-therapy in most clinical scenarios unless there is a rapid clinical progression, life-threatening visceral metastases, or there is a need for rapid symptom or disease control, where combination chemotherapy is preferred ${ }^{25}$.

Based on the results of low toxicity of weekly docetaxel compared with 3-weekly, weekly docetaxel was chosen as a sequential single agent ${ }^{26}$.

The ORR was $42.85 \%$ before randomization and increased to $56.3 \%$ for arm 1 and $60.0 \%$ for arm 2 which indicate further shrinking of measurable lesions. These results are in agreement with those of the study done by Ghosn et $\mathrm{a}^{15}$. They reported an ORR of $51 \%$ before randomization and an increase to 56 and $71 \%$ in the NavCap and the NavCap followed by docetaxel arms, respectively.

Although the ORR was higher in the docetaxel containing arm in the current study, the difference did not reach statistical significance; probably due to the relatively small sample size. Higher response in the docetaxel arm may be due to the higher percentage of patients $(53 \%)$ who had PR before its administration and more than half of patients had one metastatic site. In addition, there was no statistical significant difference in toxicity, TTP and OS between the two arms. These results are in agreement with the results of the study done by Ghosn et $\mathrm{al}^{15}$.

In conclusion, the results of the present study suggests that combination therapy regimens NavCap and the NavCap followed by weekly docetaxel regimens are well-tolerated and effective in Egyptian women with HER-2 negative MBC. A future research is needed to define patients who may benefit from a combination therapy or combination regimen followed by sequential single agent therapy.

\section{REFERENCES}

1. Colditz GA and Bohlke K. Priorities for the primary prevention of breast cancer. CA Cancer J Clin. 2014;64: 186-194.

2. Chen W, Zheng R, Zhang S, et al. Reports of cancer incidence and mortality in China 2010. Ann Transl Med. 2014;2:61.

3. Dong X, Alpaugh KR and Cristofanilli M. Circulating tumor cells (CTCs) in breast cancer: a diagnostic tool for prognosis and molecular analysis. Chin J Cancer Res. 2012;24:388-398.

4. Jung SY, Serika SM, Linkov F, et al. The effects of delays in treatment for breast cancer metastases on survival. Breast Cancer Re Treat. 2011;130:953-964.
5. Palmeri L, Vaglica M and Palmeri S. Weekly docetaxel in the treatment of metastatic breast cancer. Ther Clin Risk Manag. 2008;4:1047-1059.

6. Gogineni K and DeMichele A. Current approaches to management of Her2-negative metastatic breast cancer. Breast Cancer Res. 2012;14: 205.

7. Zelek L, Barther S, Riofrio M, et al. Weekly vinorelbine is an effective palliative regimen after failure with anthracyclines and taxanes in metastatic breast carcinoma. Cancer. 2001;92: 2267-2272.

8. NCI Drug Dictionary [http//www.cancer.gov/drugdictionary].

9. Molina-Garrido MJ, Mora-Rufete A and Guillen-Ponce C. Oral chemotherapy in elderly women with metastatic breast cancer. Anticancer Agents Med Chem. 2014;14: 665-672.

10. Ghosen M, Kattan J, Farhat F, et al. Phase II trial of capecitabine and vinorelbine as first-line chemotherapy for metastatic breast cancer patients. Anticancer Res. 2006;26:2451-2456.

11. Estevez LG, Batista N, Sanchez-Rovira P, et al. A phase II study of capecitabine and vinorelbine in patients with metastatic breast cancer pretreated with anthracyclines and taxanes. Clin Breast Cancer. 2008;8:149-154.

12. Ghersi D, Willson ML, Chan MM, et al.Taxane-containing regimens for metastatic breast cancer. Cochrane Database Syst Rev. 2015;6:CD003366.

13. Tabernero J, Climent MA, Lluch A, et al. A multicentre, randomized phase II study of weekly or 3-weekly docetaxel in patients with metastatic breast cancer. Ann Oncol. 2004; 15:1358-1365.

14. Ghosen M, Kattan J, Farhat F, et al. Sequential vinorelbinecapecitabine followed by docetaxel in advanced breast cancer: long-term results of a pilot phase II trial. Cancer Chemother Pharmacol. 2008;62:11-18.

15. Ghosen M, Aftimos P, Farhat F, et al. A phase II randomized study comparing navelbine and capecitabine (Navcap) followed either by Navcap or by weekly docetaxel in the first-line treatment of HER-2/neu negative metastatic breast cancer. Med Oncol. 2011; 28:S142-S151.

16. Brenner H, Gondos A and Arndt V. Recent major progress in long-term cancer patients survival disclosed by modeled period analysis. J Clin Oncol. 2007;25:3274-3280.

17. Jemal A, Center MM, DeSantis, et al. Global pattern of cancer incidence and mortality rates and trends. Cancer Epidemiol Biomarkers Prev. 2010;19:1893-1907.

18. European School of Oncology (ESO)-MBC Task Force. Metastatic breast cancer. Recommendations proposal from the European school of oncology (ESO)-MBC task force. Breast 2007; 16 (1): 9-10.

19. Stockler M, Wikeken NR, Ghersi D, et al. Systematic reviews of chemotherapy and endocrine therapy in metastatic breast cancer. Cancer Treat Rev. 2000;26:151-168.

20. Cardoso F, Bedard PL, Winer EP et al. International guidelines for management of metastatic breast cancer: combination vs sequential single-agent chemotherapy. J Natl Cancer Inst. 2009;101:1174-1181.

21. Carrick S, Parker S, Thornton CE, et al. Single agent versus combination chemotherapy for metastatic breast cancer. Cochrane Database Rev. 2009;2: CD003372.

22. El-Sadda W, Abdel Halim II, Abdel Aziz M, et al. Phase II study of vinorelbine and capecitabine as first-line 


Vol. $11 \mid$ No. 1-2 2015

treatment for metastatic breast cancer. J Clin Oncol. 2013; 31 (suppl; abstr e 12004).

23. Tawfik H, Rostom $\mathrm{Y}$ and Elghazaly H. All-oral combination of vinorelbine and capecitabine as firstline treatment in HER2/Neu-negative metastatic breast cancer. Cancer Chemother Pharmacol. 2013;71:913-919.

24. $\mathrm{Vu} \mathrm{T}$, Ellard S, Speers CH, et al. Survival outcome and cost-effectiveness with docetaxel and paclitaxel in patients with metastatic breast cancer: a population-based evaluation. Ann Oncol. 2008;19:461-464.

\section{NavCap vs. NavCap+docetaxel in met. Her2 -ve breast ca.}

25. Cardoso F, Costa A, Norton L et al. First international consensus guidelines for advanced breast cancer (ABC 1). Breast. 2012;21:242-252.

26. Rivera E, Mejia JA, Arun BK, et al. Phase 3 study comparing the use of docetaxel on an every-3-week versus weekly schedule in the treatment of metastatic breast cancer. Cancer. 2008;112:1455-1461. 